\title{
Systèmes d'aquifères profonds dans le bassin molassique du sud-ouest de l'allemagne
}

\author{
Analyse hydrogéologique approfondie servant de base \\ à un futur management quantitatif et qualitatif
}

\author{
par R. Watzel et B. Bertleff \\ Geologisches Landesamt Baden-Württemberg, Freiburg i. Br., R.F.A.
}

\section{E INTRODUCTION}

On utilise beaucoup dans le bassin molassique du sud-ouest de l'Allemagne, au point de vue balnéologique et géothermique, les nappes aquifères profondes composées des couches du Tertiaire (Molasse) et de l'aquifere karstique du Malm (Jurassique supérieur) sous-jacent.

Pour la conception et le fonctionnement des installations géothermiques, il faut que le système d'écoulement, les quantités d'eaux souterraines disponibles ainsi que leur capacité calorifique soient connus.

C'est dans cette intention que le Ministère de la recherche et de la technologie ainsi que la Communauté Européenne ont soutenu des projets. Le but de ces recherches était d'analyser les conditions géologiques, hydrogéologiques, géochimiques et isotopiques de l'aquifère profond (Bertleff et al., 1987, 1988 ; Prestel, 1989 ; Stober, 1984). Dans l'aquifère karstique thermal du Malm, on devait analyser les paramètres nécessaires aux modélisations hydrodynamiques et géothermiques.

L'exploitation de l'aquifère tertiaire (Molasse continentale supérieure et Molasse marine supérieure) ainsi que de l'aquifere karstique thermal dure depuis plus de dix ans. Dans certains endroits on peut constater de légers changements dans la. composition chimique et isotopique des eaux. Ces changements, mineurs au début, donnent des renseignements importants sur le développement futur hydraulique et hydrochimique de la zone d'influence des forages profonds et de l'ensemble du système. Ceci a également des répercussions sur les possibilités d'utilisation géothermique.

Pour dépister précocement des modifications hydrauliques et hydrochimiques liées aux prélèvements, le Service Géologique du Bade-Württemberg a entamé un programme de recherche à long terme. Il sera réalisé en collaboration avec

The sandstone basin of the south of Germany is an area where the geothermic exploitation is intensive. The well-working of these equipments require good attainments about the quantities of available water in the aquifer stratum and about the geothermic conditions. That is the reason why the west part of the basin has been largely prospected for almost twenty years. Hydraulic, hydrochimic and isotopic studies have shown that two circulation zones have to be differemiated. Very different replacement conditions are represented in the latter. The possibitities of geothernic exploitations are detemined by the ratio of the paleopleistocene aquiferous stratum and of the recently formed one. This study handles above all with the results of the hydrochimic and isotopic exams and with the issue of the renewal of the aguiferous stratum. The possible consequences for the furure use of the stratum will be outlined. 
l'IAEA (Nations Unies) de Vienne, I'Institut pour l'hydrologie du GSF de Munich et la firme Hydroisotop GmbH de Schweitenkirchen. Une gestion de la nappe aquifère apparaît nécessaire.

La région à étudier est située dans la partie ouest des Préalpes du sud de l'Allemagne (fig. 1) et comprend une superficie d'à peu près $4600 \mathrm{~km}^{2}$. Le Malm recouvert de molasse constitue environ $3 / 4$ de cette région. La partie restante se compose de Malm karstique affleurant. La température moyenne annuelle varie entre 8,5 et $9,0{ }^{\circ} \mathrm{C}$ à une altitude de 500 à 800 mètres. Les précipitations annuelles moyennes sont de $800 \mathrm{~mm}$. Pour le Malm karstique affleurant, on estime que la recharge de l'aquifère est de $10 \mathrm{I} / \mathrm{s}$ au $\mathrm{km}^{2}$. Elle diminue de 1,0 à $0,1 \mathrm{l} / \mathrm{s}$ au $\mathrm{km}^{2}$ lorsque la couverture de couches du Tertiaire et du Quaternaire augmente d'épaisseur.

\section{CONDITIONS GÉOLOGIQUES ET HYDROGÉOLOGIQUES}

La région étudiée se trouve dans la partie nord du bassin molassique. Le bassin est limité au nord-ouest en bordure du Danube par le plateau de Malm des Alpes souabes. Ce plateau se compose essentiellement de calcaires, qui plongent vers le Sud sous les formations tertiaires et quaternaires et forment le socle du bassin molassique (fig. 2 et 3 ).

Les formations sédimentaires apparaissent au nord des Alpes souabes et reposent elles-mêmes sur le socle cristallin. Les formations tertiaires sont en grande partie recouvertes par des dépôts glaciaires. Les couches pendent généralement de 1,5 à $2^{\circ}$ en direction du Sud-Est, le pendage augmente en bordure des Alpes.

De nombreuses failles de direction parallèles au bassin affectent l'ensemble des couches jusqu'au sommet du Tertiaire. Le rejet des failles est de 50 à 200 mètres (fig. 2 et 3). Ces failles ont une grande importance pour la circulation des eaux souterraines dans le bassin.

L'épaisseur complète du Malm atteint 550 mètres. Dans le secteur recouvert de molasse, les couches du Kimmeridgien moyen et supérieur (ki 2-3), du Tithonique (tiL) profond constituent le véritable aquifère. Il se compose de dolomies et de calcaires dolomitiques à gros grain, poreux et fortement fissurés. Son épaisseur est d'environ 250 mètres. Les couches au-dessus et au-dessous de l'aquifere sont plus ou moins imperméables. A l'intérieur de l'aquifère ki2-tiL, ce sont essentiellement les parties comprenant des dolomies de diagénèse précoce et des dolomies dédolomitisées caverneuses qui sont aquifères. C'est pourquoi l'épaisseur utile de l'aquifère proprement dit est seulement de 20 à 60 mètres. La porosité efficace est de 2.5 à $3 \%$. La partie restante de la série carbonatée ki2-tiL, qui est beaucoup plus épaisse, fonctionne comme un réservoir secondaire.

Au sein de l'aquifère karstique du Malm l'intensité de la karstification faiblit du Nord-Ouest vers le Sud-Est. On peut considérer la ligne de changement de faciès entre les faciès souabes au nord et les faciès helvétiques au sud comme étant la frontière sud de l'aquifère du Malm. Les calcaires des faciès helvétiques du Quintner sont peu perméables.

En regardant la carte des isopièzes du potentiel hydraulique (fig. 1), on peut en déduire deux directions principales d'écoulement dans l'aquifère karstique du Malm. Un écoulement sud se dirige vers le lac de Constance avec un gradient

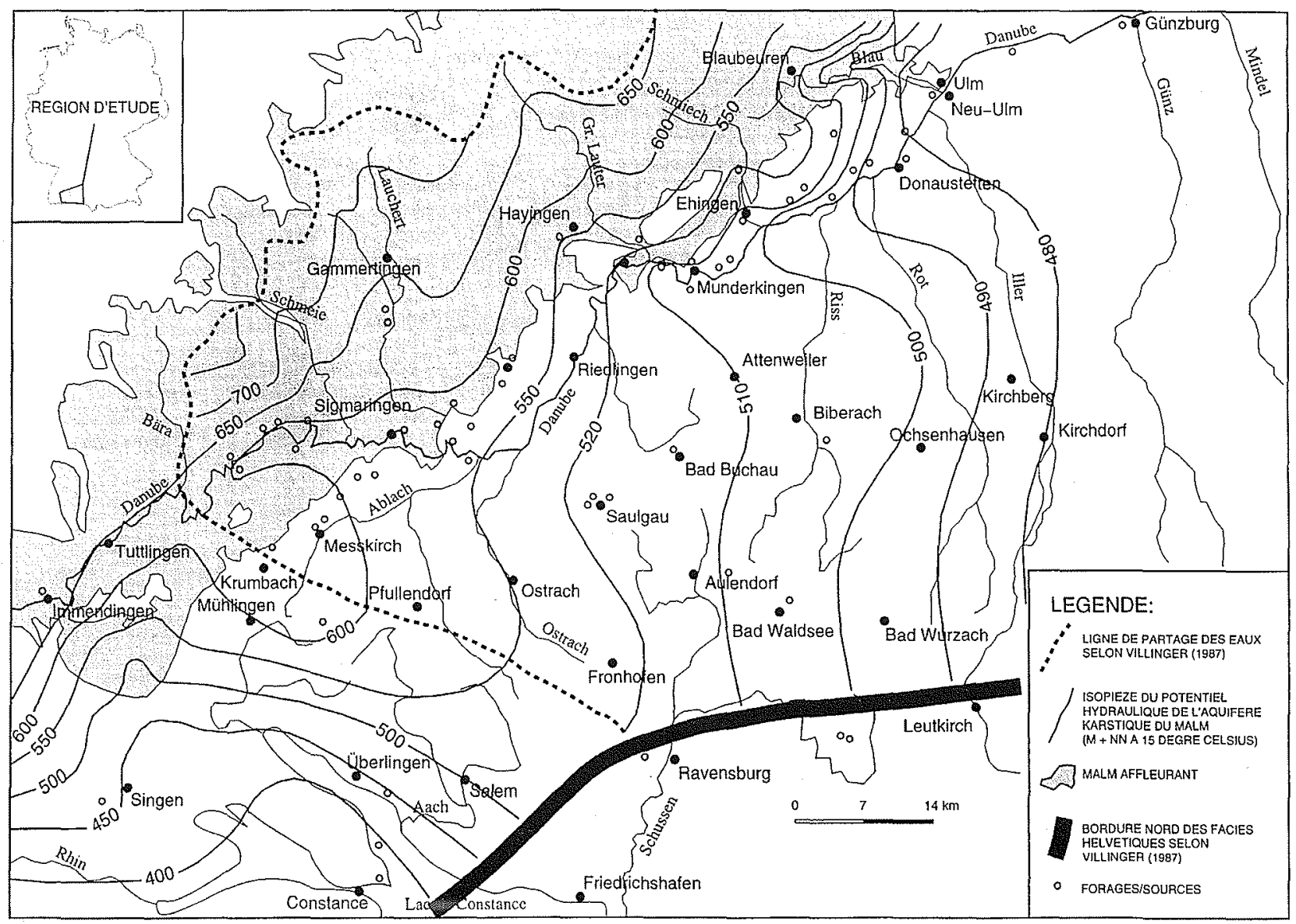




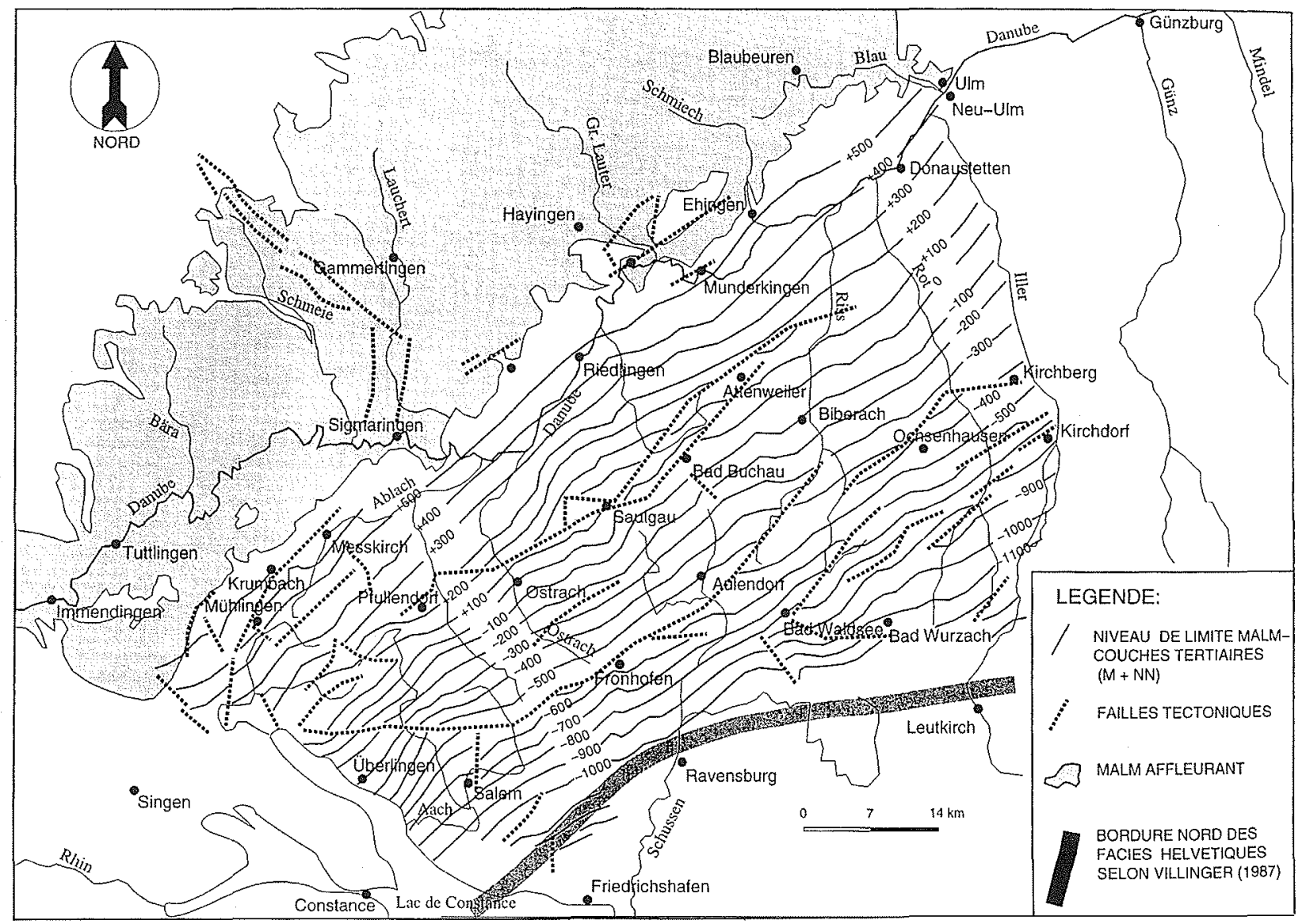

de 2 à $3 \%$. Un écoulement est se dirige avec un gradient plat vers le fossé de drainage régional du Danube. L'écoulement vers l'Est est plus important au point de vue hydrogéologique. La ligne de partage des eaux souterraines karstique traverse la limite nord des Alpes souabes en passant sous le Danube jusqu'à la limite de faciès à Ravensburg. On la nomme aussi ligne de partage des eaux karstique européenne, car elle délimite les bassins-versants du Rhin et du Danube.

Le potentiel hydraulique de l'eau thermale dans le Malm supéricur est plus faible que celui de la Molasse marine supérieure sus-jacente. La différence de pression atteint 50 mètres au centre de bassin et environ 20 mètres sur sa bordure sud.

Dans le Malm supérieur, la température de l'eau thermale qui s'élève à $20^{\circ} \mathrm{C}$ à la hauteur du Danube, augmente régulièrement en direction du centre du bassin avec le pendage des couches (fig. 3). On atteint les températures les plus élevées d'environ $80^{\circ} \mathrm{C}$, le long de la limite des faciès helvétiques.

\section{CARACTÉRISTIQUES HYDROCHI- MIQUES ET ISOTOPIQUES}

De nombreuses études hydrochimiques et mesures isotopiques ont aidé à la compréhension du principe et du fonctionnement des systèmes de circulation dans le bassin molassique. Les isotopes ${ }^{2} \mathrm{H},{ }^{3} \mathrm{H},{ }^{13} \mathrm{C},{ }^{14} \mathrm{C},{ }^{18} \mathrm{O},{ }^{85} \mathrm{Kr}$ et les gaz rares ont été analysés.
Au vu des résultats des analyses hydrochimiques et isotopiques, on peut déduire la présence de deux zones de circulation dans l'aquifère karstique du Malm :

- Une zone nord, située au bord du bassin, composée d'eau du type $\mathrm{Ca}-\mathrm{Mg}-\mathrm{HCO}_{3}$. Ses teneurs élevées en ${ }^{2} \mathrm{H}$ et ${ }^{18} \mathrm{O}$ ainsi que les températures des gaz rares indiquent une recharge dans des conditions climatiques holocènes.

- Une zone sud située au cœur du bassin et composée d'eau de type $\mathrm{Na}-\mathrm{Ca}-\mathrm{HCO}_{3}-\mathrm{Cl}$ (échange d'ions) qui venant de l'aquifère susjacent de la molasse, s'infiltre dans l'aquifere karstique du Malm. Ses teneurs pauvres en ${ }^{18} \mathrm{O}$ et ses températures de gaz rares suggèrent une recharge sous des conditions climatiques pléistocènes.

Si l'on représente les teneurs en alcalins $(\mathrm{Na}+\mathrm{K})$ et celles en $\delta^{18} \mathrm{O}$ sur une carte (fig. 4), les deux paramètres montrent la répartition des deux zones de circulation. Entre les eaux holocènes au Nord, de type Ca- $\mathrm{Mg}-\mathrm{HCO}_{3}$, et les eaux pléistocènes de type $\mathrm{Na}-\mathrm{Ca}-\mathrm{HCO}-\mathrm{Cl}$ s'est formée une étroite zone de transition.

On peut également distinguer sur le graphique des données $\delta^{18} \mathrm{O}$, en fonction des teneurs en ${ }^{14} \mathrm{C}$, la limite des deux zones des circulation (fig. 5). Il en est de même pour la relation des données $\delta^{18} \mathrm{O}$ en fonction des températures des gaz rares (fig. 5). La figure 5 montre une diminution importante des données $\delta^{18} \mathrm{O}$ en dessous d'une teneur en ${ }^{14} \mathrm{C}$ de $10 \%$ de l'actuel. Une teneur en ${ }^{14} \mathrm{C}$ de $10 \%$ de l'actuel correspond à peu près à la limite dans le temps entre l'Holocène et le Pléistocène. La variabilité des données en $\delta^{18} \mathrm{O}$ pour une forte teneur en ${ }^{14} \mathrm{C}$ peu être interprétée comme la conséquence des effets climatiques récents. Il s'agit ici de l'effet d'altitude, de l'effet continental et des fluctuations saisonnières. Dans la zone de transition, on trouve des eaux mélan- 

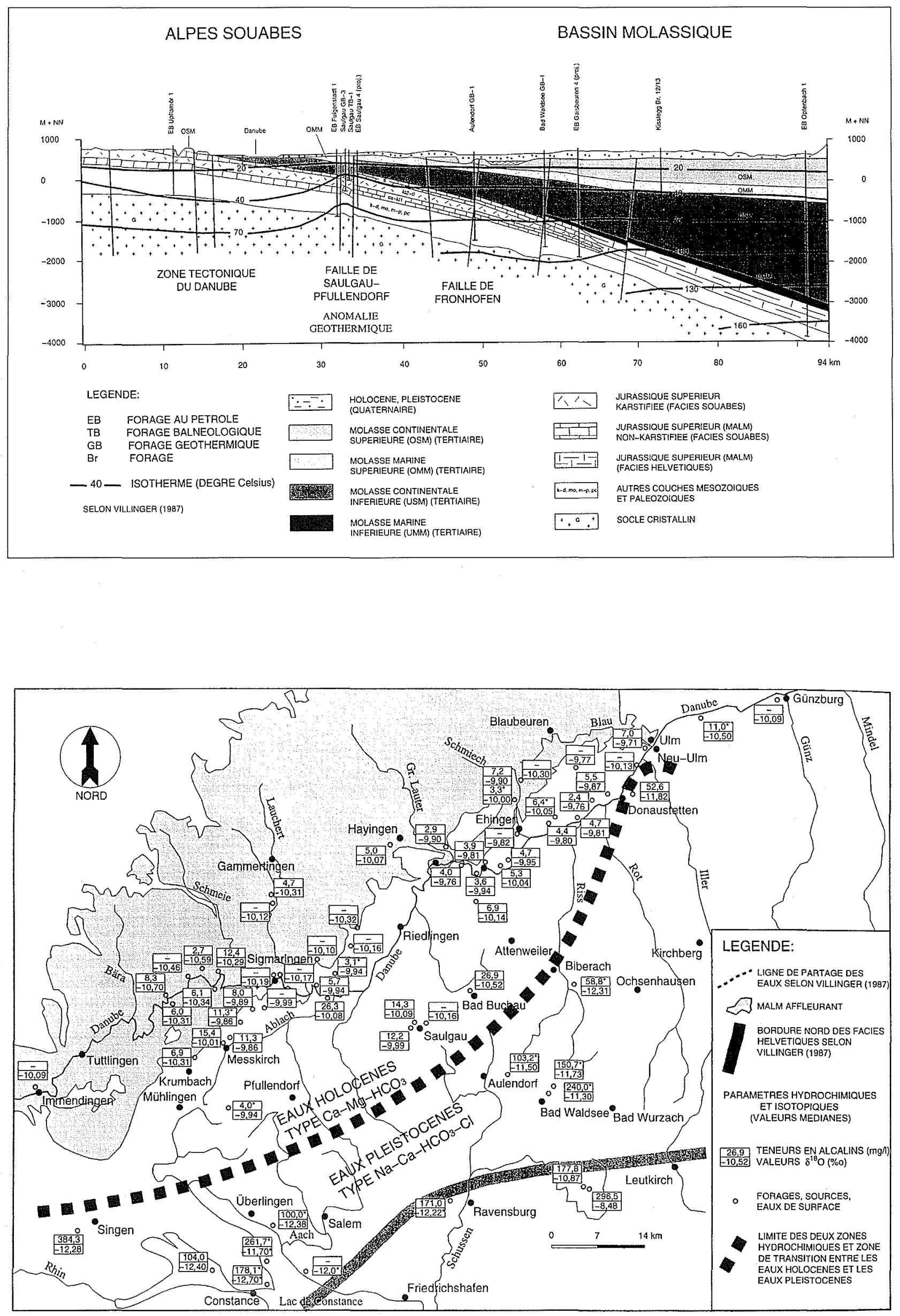
gées qui présentent une proportion variable d'eaux de composition pléistocène.

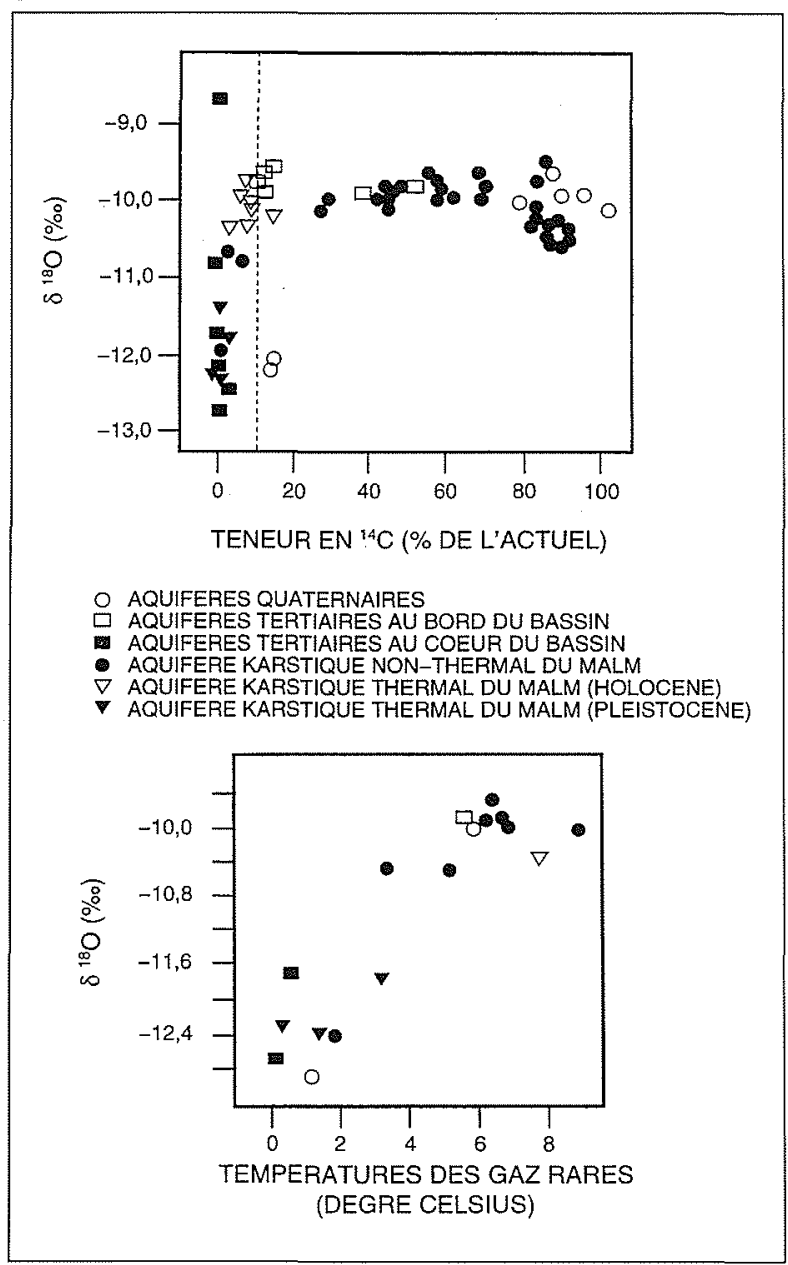

Les teneurs en gaz rares sont une mesure des températures moyennes du sol lors de la recharge de l'aquifère ( $\ll$ températures des gaz rares »). La figure 5 montre la relation entre les teneurs en gaz rares et les données $\delta^{18} \mathrm{O}$. Les valeurs faibles en $\delta^{18} \mathrm{O}$ correspondent à des températures faibles de recharge, des valeurs élevées en $\delta^{18} \mathrm{O}$ à des températures élevées de recharge. Cette relation qualitative est confirmée et quantifiée par les températures des gazs rares. Les eaux du cœur du bassin se sont formées dans des conditions climatiques plus froides, celles situées sur le bord du bassin dans des conditions climatiques plus récentes. Avec l'aide de l'âge ${ }^{14} \mathrm{C}$ des eaux souterraines, on a estimé pour la partie nord de l'aquifère karstique du Malm des vitesses de circulation de 2 mètres par an. Des valeurs analogues sont également obtenues par les essais hydrauliques.

Les données $\delta^{18} \mathrm{O}$, les teneurs en ${ }^{14} \mathrm{C}$ ainsi que les températures des gaz rares montrent que les eaux des aquifères molassiques qui se trouvent à l'intérieur du bassin, sont aussi d'âge pléistocène. Ceci confirme l'hypothèse selon laquelle la recharge de l'aquifère karstique du Malm provient de l'infiltration des eaux des aquifères tertiaires.

Le résidu sec des eaux thermales de l'aquifère karstique du Malm est de 450 à $950 \mathrm{mg} / \mathrm{l}$.

\section{O CARACTÉRISTIQUES HYDRODYNA- MIQUES}

Dans le secteur étudié on a testé l'aquifère karstique du Malm affleurant et celui recouvert de molasse en faissant de nombreux essais de pompage. Dans certains cas, des essais de traçage ont contribué à l'établissement des paramètres hydrodynamiques. Au vu des résultats, on peut également partager l'aquifère karstique en deux zones :

- une zone nord, proche du bord du bassin avec des transmissivités $\mathrm{T}=3,8-5,4 * 10^{-3} \mathrm{~m}^{2} / \mathrm{s}$,

- une zone sud, située au centre du bassin avec des transmissivités nettement moindres $T=1,2-6,5 * 10^{-4} \mathrm{~m}^{2} / \mathrm{s}$.

Dans la partie proche des faciès helvétiques, on n'a pas pu mesurer de perméabilité notable dans le Malm supérieur. Au moyen des essais de traçage, on a estimé la porosité efficace à $2,7 \%$ dans la partie nord de l'aquifère karstique du Malm.

\section{RECHARGE DE LA NAPPE}

La composition hydrochimique et isotopique des aquifères montre que leur recharge suit des mécanismes divers dans les différents secteurs du bassin. On doit différencier une recharge holocène sous des conditions climatiques récentes d'une recharge pléistocène dans des conditions plus froides.

\subsection{Recharge récente de l'aquifère karstique du Malm}

La recharge se produit grâce à des précipitations récentes dans le secteur du Malm karstique affeurant et peu recouvert. Par suite des conditions de circulation, cette eau souterraine ne peut que s'écouler très faiblement vers le milieu du bassin. La recharge récente dans le centre du bassin se fait par infiltration à travers les couches de molasse tertiaire. II se produit une infiltration de plus au moins $0,1 \mathrm{l} / \mathrm{s}$ au $\mathrm{km}^{2}$ suivant le potentiel hydraulique.

\subsection{Hypothèse de recharge pléistocène des aquifères de la Molasse et du Malm karstique}

Dans les séquences stratigraphiques du Quaternaire, on note deux conditions climatiques extrêmes. D'un côté, des périodes de climat tempéré avec des conditions climatiques analogues à celles d'aujourd'hui, d'un autre côté, des périodes beaucoup plus froides, lorsque de grandes surfaces étaient recouvertes de glaciers. Dans les régions périglaciaires, la surface de la terre était gelée en permanence.

Pendant la période glaciaire, la recharge de l'aquifère dans les régions affectées par le «Permafrost» n'était pas possible. Il s'agissait des régions d'affleurement du Malm et de la Molasse marine supérieur, en bordure nord du bassin molassique. Sous la calotte glaciaire des secteurs importants n'étaient pas gelés.

La recharge de l'aquifère ne peut avoir eu lieu que sous la glace, vraisemblablement dans des chenaux et bassins surcreusés glaciaires. On peut admettre que les potentiels hydrauliques pléistocènes se trouvaient entre le bord de la calotte glaciaire et la surface du glacier (fig. 6).

Dans le secteur du glacier rhénan, la recharge de l'aquifère de la Molasse marine supérieure a eu lieu essentiellement dans le bassin du lac de Constance. En raison de son potentiel hydraulique élevé, l'aquifère de la Molasse marine supérieure a pu s'infiltrer dans l'aquifère karstique du Malm à travers les couches peu perméables de la Molasse conti- 


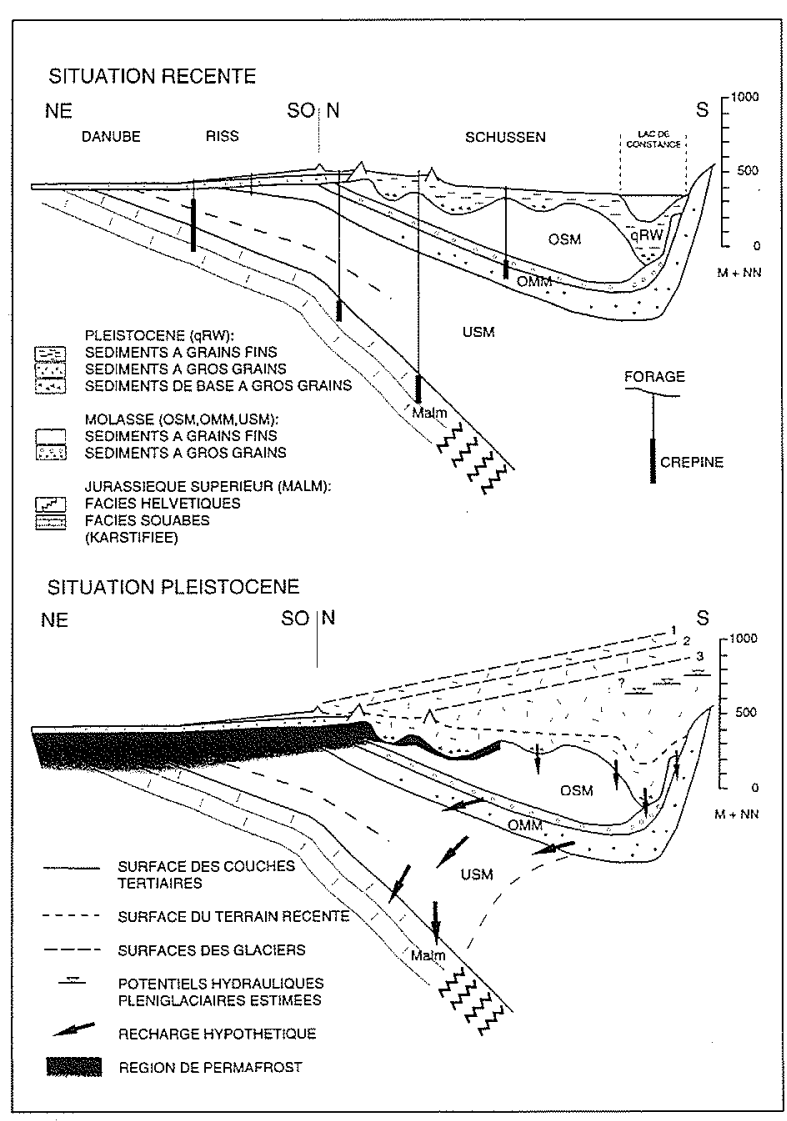

nentale inférieure. Ceci s'est produit essentiellement dans des zones de failles rectoniques qui ont une grande perméabilité de fracture. La circulation descendante doit n'avoir lieu que lorsque les exutoires naturels du Malm karstique à l'Est près de Ratisbonne ont été libres de gel permanent. Ceci a vraisemblablement été induit par l'écoulement d'eau tempérée du Malm en provenance de l'intérieur du bassin. A la même période un écoulement latéral de l'aquifère dans la Molasse marine supérieure n'était pas possible. Les régions affleurant au Nord (Périglaciaire) étaient gelées en permanence. Le Malm karstique sous-jacent était le drainage du système hydraulique de la Molasse marine supérieure, influencé par les glaces (Bertleff et al. 1993).

Si tenté que cette supposition soit juste, les nappes aquifères du centre du bassin font partie d'un système hydraulique transitoire. Les potentiels hydrauliques de ce système diminuent depuis le Pléistocène. Cette hypothèse de recharge est actuellement en cours d'étude au moyen d'un modèle numérique.

\section{RÉ RUMÉ ET CONCLUSIONS}

Dans le Malm qui a une épaisseur allant jusqu'à 550 mètres, c'est la succession de calcaires et de dolimies du Kimméridgien moyen et supérieur (ki 2-3) et du Tithonique profond (tiL) qui forme le véritable aquifère. Son épaisseur varie entre 75 et 250 mètres. Dans la série ce sont essentiellement les parties comprenant des dolomies de diagénèse précoce ou caverneuses qui sont aquifères. Les zones des circulations principales ont entre 20 et 50 mètres d'épaisseur. Le reste de la série carbonatée fonctionne comme un réservoir secondaire. C'est grâce à une karstification intense dans le secteur proche de la bordure du bassin que la perméabilité et la circulation sont élevées. Au Sud de la Iimite des faciès helvétiques, la perméabilité du Malm est beaucoup plus faible ou inexistante.

Du point de vue hydraulique, hydrochimique et isotopique on peut diviser l'aquifère karstique du Malm en deux parties.
Les données hydrochimiques et isotopiques montrent, dans la zone proche du bord du bassin la présence d'une nappe aquifềre récente. Dans le centre du bassin, la nappe aquifère est d'origine pléistocène. Un échange d'ions a eu lieu aú cours des infiltrations dans les couches de molasse susjacentes.

La différence de potentiel hydraulique entre l'aquifère du Malm et celui de la couverture molassique indique que la recharge récente est minime dans le centre du bassin. Cette nappe aquifère appartient vraisemblablement à un système hydraulique transitoire. Si la recharge à l'intérieur du bassin a eu lieu essentiellement au Pléistocène, on doit considérer l'aquifère karstique du Malm à cet endroit comme un gisement fossile non renouvelable. Une extraction de ce gisement aurait pour conséquence de faire affluer les eaux de la bordure du bassin. La nappe aquifere de bordure du bassin est de recharge récente et présente une autre composition hydrochimique. Des changements dans la composition hydrochimique de la nappe aquifêre du centre du bassin et dans ses propriétés géothermiques en seraient la conséquence.

Dans cette situation, il serait nécessaire de concevoir un plan de gestion des eaux souterraines des aquifères du Tertiaire et du Jurassique supérieur (Malm). En ce moment des travaux préparatifs sont en cours. On est en train de procéder à des études hydrochimiques et isotopiques à long terme dans les différents forages. Suivant la situation hydrogéologique, des paramètres spécifiques vont être examinés dans les différents forages. Des modélisations numériques sont nécessaires pour des prévisions hydrauliques et géothermiques quantitatives. Un modèle stationnaire de l'ensemble du bassin molassique est déjà prêt. Un modèle transitoire détaillé de la partie ouest est en cours d'élaboration.

\section{VII $\mathbf{0}$ RÉFÉRENCES}

11] BERTLEFF, B. (1986) : Das Strömungssystem der Grundwässer im Malm-Karst des Westteils des süddeutschen Molassebeckens. - Abh. Geol. Landesamt Baden-Würtemberg, 12 : 1 271 ; Freiburg i. Br.

12] BERTLEFF, B., HAMMER, W., JOACHIM, H., KOZIOROWSKI, G., STOBER, I., STRAYLE, G., VILLINGER, E. \& WERNER, J. (1987) : Hydrogeothermiebohrungen in BadenWürtemberg. Eine Ubersicht. - Z. dt. geol. Ges., $138: 41$ 1423 ; Hannover.

[3] BERTLEFF, B., JOACHIM, H., KOZIOROWSKI, G., LEIBER, J., OHMERT, W., PRESTEL, R., STOBER, I., STRAYLE, G., VILLINGER, E. \& WERNER, J. (1988) : Ergebnisse der Hydrogeothermiebohrungen in Baden-Württemberg. - Jh. geol. Landesamt Baden-Wüttemberg, $30: 27$ 116 ; Freiburg i. Br..

[4] BERTLEFF, B., ELLWANGER, F., SZENKLER, C., EICHINGER, L., TRIMBORN, P. \& WOLFENDALE, N. (1993) : Interpretation of hydrochemical and hydroisotopical measurements on paleogroundwaters in Oberschwaben, South German Alpine foreland, with focus on quartemary geology. - Proc. Symp. Vienna 19-23 April 1993, IAEA-SM-329/63 ; Vienna.

15] PRESTEL, R. (1989) : Der Isotopengehalt von Kluft-Calciten aus dem Malm-Kern der Bohrung Saulgau GB-3. - Abh. geol. Landesamt Baden-Wurttemberg, 13 : 161-180 ; Freiburg i. Br.

16] STOBER, I. (1984) : Hydrogeologische Untersuchungen in Festgesteinen Suidwestdeutschlands mit Hilfe von Pump- und Injektionsversuchen. - Diss. Univ. Freiburg, 119 S. ; Freiburg i. $\mathrm{Br}$.

Nous adressons nos remerciements à Mme M.-H. Ronneburger du Service Géologique du Bade-Württemberg et à M. Philippe Elsass, Directeur du Service Régional d'Alsace pour leur aide précieuse dans la traduction de ce dossier. 